\title{
On Resilient Control of Nonlinear Systems under Denial-of-Service
}

\author{
Claudio De Persis
}

\author{
Pietro Tesi
}

\begin{abstract}
We analyze and design a control strategy for nonlinear systems under Denial-of-Service attacks. Based on an ISS-Lyapunov function analysis, we provide a characterization of the maximal percentage of time during which feedback information can be lost without resulting in instability of the system. Motivated by the presence of a digital channel we consider event-based controllers for which a minimal intersampling time is explicitly characterized.
\end{abstract}

\section{INTRODUCTION}

Motivated by interest in the analysis and control of critical infrastructures such as power networks, supply chains and transportation systems, recent years have witnessed increasing research interests in large-scale engineered systems. To achieve the prescribed control goal, these systems require exchange of information that often occurs in digital form. In turn this has triggered interest in control over communication channels. One of the topics that has stimulated broad interest is the so-called event-based control ([19]) in which sampling times are designed in real-time with the ultimate goal of saving communication resources while still guaranteeing the control goal. Event-based control has found fertile ground also in the area of cooperative control; e.g., see [18], [4].

A natural research question raises when dealing with control over a communication channel: whether or not stability properties and performance are preserved in the presence of loss of feedback information. This loss of information could be due not only to malfunctioning but also to malicious actions by an adversarial entity [1], [12]. In the latter case, the assumption on the kind of information loss should be kept to a minimum since intelligent adversaries might not follow e.g. any statistical pattern. This aspect is in contrast with other work where the loss of information is mainly due to the unreliability of the communication channel [17].

Several contributions to the topic of stability/stabilization in the presence of adversarial entities have been reported in the last few years, with main emphasis on the so-called Denial-of-Service (DoS), a class of attack strategies primarily intended to affect the timeliness of information exchange [3]. In [1], the authors address the problem of security constrained optimal control for discrete-time linear systems in which packets may be jammed by a malicious adversary, and the goal is to find optimal control and attack strategies assuming a maximum number of jamming actions over a prescribed (finite) control horizon. A very similar scenario

C. De Persis and Pietro Tesi are with Faculty of Mathematics and Natural Sciences, University of Groningen, the Netherlands, $\{c \cdot d e \cdot p e r s i s, p . t e s i\}$ @rug.nl. This work is partially supported by the Dutch Organization for Scientific Research (NWO) under the auspices of the project QUICK (QUantized Information Control for formation Keeping). is considered in [10], where the problem of stabilizing a discrete-time linear system under DoS is casted as a dynamic zero-sum game. An interesting alternative scenario is addressed in [8], where the authors consider the problem of stability under periodic DoS for linear sampled-data systems under state-feedback. The idea there is to identify the jamming signal so as to restrict the information exchange to the time intervals where no DoS occurs. This approach has been then extended in [7] by considering energy-constrained, but otherwise unknown DoS attacks.

In [5], [6], we addressed afresh the problem of stability under energy-constrained, but unknown, DoS attacks within the framework of linear sampled-data systems under statefeedback. The analysis differs from the one in [7], [8] since the goal is not to identify the jamming signal; rather, the goal is to determine if stabilization is possible assuming only a bound on the fraction of the time the jammer is active. The considered approach, inspired by [19], consists in a suitable logic that determines in real-time the frequency of controller updates (the sampling times) depending on the DoS occurrence. In particular, it enjoys the following features:

i) It ensures global exponential stability of the closed-loop system whenever the intervals over which communication is possible are predominant with respect to the intervals over which communication is denied;

ii) It allows for the state-feedback matrix to be designed in accordance with any control design method, robustness against DoS being achieved thanks to the sampling logic;

iii) It is resilient since the sampling rate varies depending on the DoS occurrence;

iv) It allows for an explicit characterization of convergence rate, minimal inter-sampling time, and ratios between the "active" and "sleeping" periods of DoS which do not destroy closed-loop stability;

v) It is flexible enough so as to allow the designer to choose from several implementation options that can be used to trade-off performance vs. communication resources.

The objective of this paper is to initiate the investigation of similar ideas for nonlinear systems. Although we follow the line of arguments of [5], [6], a few of the steps we take are very peculiar to nonlinear systems, making the extension far from straightforward and deserving attention on its own right. It is shown that under certain additional conditions, which are basically needed to avoid finite-escape times phenomena during DoS, semi-global asymptotic stability can be still ensured. The analysis combines arguments from event-based 
control and ISS control Lyapunov functions.

The remainder of this paper is organized as follows. In Section II we introduce the framework of interest and provide an overview of the problem. In Section III, we describe the considered class of DoS attacks and provide some preliminary stability results. The main result with a characterization of the class of DoS signals under which stability is preserved is given in Section IV. In Section V, we provide a characterization of the achieved minimal intersampling rate. Section VI provides concluding remarks and outlines future research directions.

\section{FRAMEWORK AND PROBLEM OVERVIEW}

In this paper we consider nonlinear systems of the form

$$
\dot{x}=f(x, u)
$$

for which we assume the existence of a smooth state feedback $u=k(x)$ which renders the closed-loop system

$$
\dot{x}=f(x, k(x+e))
$$

ISS with respect to measurement errors $e$ in the sense that there exist a smooth function $V$ and class $\mathcal{K}_{\infty}$ functions $\alpha_{1}, \alpha_{2}, \gamma_{2}$ such that

$$
\begin{aligned}
& \alpha_{1}(\|x\|) \leq V(x) \leq \alpha_{2}(\|x\|) \\
& \nabla V(x) f(x, k(x+e)) \leq-\lambda V(x)+\gamma_{2}(\|e\|),
\end{aligned}
$$

with $\lambda>0$.

The control action is implemented via a sample-and-hold device. In a nominal situation, given a sequence of times $\left\{t_{k}\right\}, k \in \mathbb{N}$, where $t_{0}:=0$ by convention, the control action is such that

$$
u_{\text {nom }}(t)=k\left(x\left(t_{k}\right)\right), \quad \text { for all } t \in\left[t_{k}, t_{k+1}\right) .
$$

The mechanism that generates this sequence of times will be specified in the sequel. By nominal situation is meant that at each time $t_{k}$ at which the actuator needs to update the control value, it correctly receives the sampled value $k\left(x\left(t_{k}\right)\right)$.

The focus of this paper is on a scenario that is different from the nominal one, namely one in which there might be times in the sequence $\left\{t_{k}\right\}$ at which the control value cannot be updated because no information regarding $k\left(x\left(t_{k}\right)\right)$ is received by the actuator. This loss of information can be caused by several factors, such as a defective communication channel or as a consequence of the action of an adversarial entity. To fix the ideas, we focus in the sequel on the latter scenario, and refer to this interruption of information transmission from the sensor to the actuator as Denial of Service or DoS.

Let $\left\{h_{n}\right\}, n \in \mathbb{N}, h_{0} \geq 0$, represent a sequence of "positive edge-triggering" times which define the time intervals at which a DoS attack is occurring. Namely, at $h_{n}$ the $n$-th DoS attack becomes active (no communication is possible from the sensors to the actuators), while at $h_{n}+\tau_{n}$, with $\tau_{n}>0$ the duration of the $n$-th DoS attack during which information transmission is not possible, the DoS attack

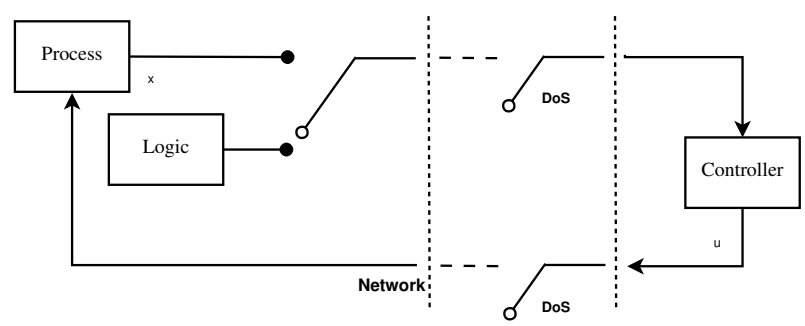

Fig. 1. Block diagram of the closed-loop system under DoS on the communication channels.

ends (communication is possible from the sensors to the actuators). In formula,

$$
H_{n}=\left[h_{n}, h_{n}+\tau_{n}[\right.
$$

represents the $n$-th DoS time-interval.

We then assume that, in the presence of DoS, the actuator generates an input that is based on the most recently received control signal. Specifically, denote the set of time-instants where communication is possible by

$$
\Theta(t):=[0, t] \backslash \bigcup_{n \in \mathbb{N}} H_{n}
$$

where $\backslash$ means relative complement. Accordingly, the control input applied to the process at each time instant can be expressed as

$$
u(t)=k\left(x\left(t_{k(t)}\right)\right)
$$

where

$k(t):= \begin{cases}-1, & \text { if } \Theta(t)=\emptyset \\ \sup \left\{k \in \mathbb{N} \mid t_{k} \in \Theta(t)\right\}, & \text { otherwise }\end{cases}$

denote the last (up to the current time) successful control update. Notice that $h_{0}=0$ implies $k(0)=-1$, which raises the question of assigning a value to the control input when communication is not possible at the process start-up. In this respect, we assume that when $h_{0}=0$ then $u(0)=0$, and we let $x\left(t_{-1}\right):=0$ for notational consistency.

The problem of interest is to find suitable control update rules, i.e. logics for generating $\left\{t_{k}\right\}_{k \in \mathbb{N}}$ that are resilient against DoS, while ensuring the existence of a minimal control update inter-execution time. The requirement of resilience calls for logics capable not only to tolerate but also to counteract the occurrence of DoS. On the other hand, requiring the existence of a minimal control update inter-execution time is fundamental in order for the control architecture to be implementable on digital platforms.

The following definitions are key for the developments of the paper.

Definition 1 System $\Sigma$, composed of (1) in closed-loop with (6) is said to have a semi-globally asymptotically stable origin if there exists a $\mathcal{K} \mathcal{L}$ function $\beta$ such that, for all $R>0$, the solution to $\Sigma$ satisfies

$$
\|x(t)\| \leq \beta(\|x(0)\|, t)
$$


for all $t \in \mathbb{R}_{\geq 0}$ and for all $x(0) \in \mathbb{R}^{n_{x}}$ such that $\|x(0)\| \leq$ $R$, where $\| \cdot \overline{\|}$ stands for Euclidean norm.

Definition 2 A control update sequence $\left\{t_{k}\right\}$ is said to occur at $a$ finite sampling rate if there exists an $\varepsilon \in \mathbb{R}_{>0}$ such that

$$
\Delta_{k}:=t_{k+1}-t_{k} \geq \varepsilon
$$

for all $k \in \mathbb{N}$.

Remark 1 It is worth pointing out that the synthesis of control laws achieving ISS is in general a nontrivial task. Nonetheless, the literature on the topic is quite vast. In particular, contributions centered around the concept of ISScontrol Lyapunov functions can be found in [9], [20], [11]; see also [21] for relevant design examples.

\section{A PREPARATORY ANALYSIS}

In this section, we make some considerations regarding the considered class of DoS attacks and provide some preliminary stability results.

\section{A. DoS attacks}

In general the uncontrolled system (1) (i.e. with $u=0$ ) might have an unstable dynamics and also exhibit finiteescape times. This suggests two facts: (i) the duration of a DOS attack cannot be arbitrarily large; and (ii) conditions on the systems and of the DoS attack must make sure that information is delivered before the occurrence of a possible finite-escape time.

Given a sequence $\left\{h_{n}\right\}$, let

$$
\Xi(t):=\bigcup_{n \in \mathbb{N}} H_{n} \bigcap[0, t]
$$

denote the total interval of DoS up to the current time, where, given an interval $I$, let $|I|$ denote its length. The following assumptions are then considered.

Assumption 1 The DoS sequence $\left\{h_{n}\right\}, n \in \mathbb{N}$, is such that $\lim _{n \rightarrow \infty} h_{n}=\infty$. Moreover, there exist constants $\kappa \in \mathbb{R}_{\geq 0}$ and $\tau \in \mathbb{R}_{>0}$ such that

$$
|\Xi(t)| \leq \kappa+\frac{t}{\tau}
$$

for all $t \in \mathbb{R}_{\geq 0}$.

Assumption 2 There exists a positive value $\mu$ such that

$$
\gamma_{2}(4 r) \leq \mu \alpha_{1}(r)
$$

for all $r \in \mathbb{R}_{\geq 0}$.

In accordance with (i), limiting the duration of DoS is necessary for closed-loop stability to be conceptually achievable. Assumption 1 captures well this prescription as it requires the existence of a bound on the fraction of time in which DoS is active. On the other hand, no conditions are imposed on the DoS "structure": first, DoS is allowed to occur aperiodically; second, the duration of any two different DoS intervals need not be equal to one another.
On the other hand, the role of Assumption 2 is that of limiting the rate of divergence of the process states during the time intervals over which the control action cannot be updated. In this connection, as will become apparent in the sequel, Assumption 2 ensures that over such intervals the rate of divergence of the process states is at most exponential, which guarantees the absence of finite-escape times phenomena. Notice that when the process dynamics are linear, Assumption 2 is always satisfied.

\section{B. Ideal sampling logic and preliminary analysis}

Given Assumptions 1 and 2, one expects that stability is not destroyed if the intervals over which communication is possible are predominant with respect to the intervals over which communication is denied. However, proving this fact is far from straightforward within the classical framework of nonlinear sampled-data systems ([15], [13], [14], [2]). Even more importantly, periodic sampling strategies hamper the possibility of increasing the closed-loop robustness by adapting the sampling rate to the DoS occurrence. As shown next, the use of aperiodic sampling strategies as introduced by [19] provides a convenient framework to work with, in terms of both ease of analysis and effectiveness.

Consider the system $\Sigma$ composed of (1) in closed-loop with (6), namely

$$
\dot{x}(t)=f\left(x(t), k\left(x\left(t_{k(t)}\right)\right), \quad t \in\left[t_{k(t)}, t_{k(t)+1}\right) .\right.
$$

Let

$$
e(t):=x\left(t_{k(t)}\right)-x(t)
$$

be the measurement error induced by the sampling, with $e\left(t_{k(t)}\right)=0$ by definition. System $\Sigma$ can be therefore written as

$$
\dot{x}(t)=f(x(t), k(x(t)+e(t))), \quad t \in\left[t_{k(t)}, t_{k(t)+1}\right) .
$$

Consider next the following ideal sampling logic. Given a sampling instant $t_{k}$ :

i) if $t_{k}$ does not belong to $\Xi(t)$, then the next sampling instant $t_{k+1}$ is defined as the infimal time larger than $t_{k}$ such that the condition

$$
\gamma_{2}(4\|e(t)\|) \leq \lambda(1-c) V(x(t)),
$$

with $c \in(0,1)$, is violated;

ii) if instead $t_{k}$ belongs to some DoS interval $H_{n}$, then the next sampling instant $t_{k+1}$ is defined as $h_{n}+\tau_{n}$.

This logic ensures that (14) holds true for all $t \in \Theta(t)$. Unfortunately, it is ideal in the sense that it cannot be implemented over digital platforms. In fact, because of the possible aperiodic nature of DoS, it is conceptually impossible to foresee when Dos will cease, and, hence, it is impossible to implement point ii). Nonetheless, at this stage of analysis, it is convenient to focus on this logic. Variants that are implementable over digital platforms will be discussed in Sections IV and V.

We are now in the position to provide a preliminary closed-loop stability analysis. The underlying idea can be 
described as follows: we decompose the time axis into the sets $\Theta(t)$ and $\Xi(t)$. Under the considered logic, (14) holds by construction over $\Theta(t)$. In turns, this ensures that (2) satisfies a dissipation-like inequality. On the other hand, over $\Xi(t)$, (14) need not hold and the closed-loop system might exhibit divergence trends. Overall, the closed-loop dynamics can be therefore viewed as those of a hybrid system, where one switches between stable and unstable modes. The idea is then to determine conditions under which the stable behavior is predominant with respect to the unstable one.

We make these considerations precise. First notice that, as an immediate consequence of (14), for all $t \in \Theta(t)$, we have

$$
\dot{V}(x(t)) \leq-c \lambda V(x)=:-\omega_{1} V(x(t)) .
$$

On the other hand, for all $t \in H_{n}=\left[h_{n}, h_{n}+\tau_{n}\right]$ for some $n \in \mathbb{N}$, we have that

$$
\dot{V}(x(t)) \leq-\lambda V(x(t))+\gamma_{2}(\|e(t)\|)
$$

We would like to bound the growth of $e(t)$ as a function of $V(x(t))$. Recall that

$$
\|e(t)\| \leq\left\|x\left(t_{k\left(h_{n}\right)}\right)-x(t)\right\| \leq\left\|x\left(t_{k\left(h_{n}\right)}\right)\right\|+\|x(t)\| .
$$

To bound $\left\|x\left(t_{k\left(h_{n}\right)}\right)\right\|$, we need to introduce an appropriate lemma.

Lemma 1 Under Assumption 2, the state $x(t)$ of system (1) under the control update rule (14) and sample-and-hold feedback control (6) satisfies

$$
\begin{aligned}
\left\|x\left(t_{k\left(h_{n}\right)}\right)\right\| \leq & \frac{1}{4} \gamma_{2}^{-1}\left(\lambda(1-c) V\left(x\left(h_{n}\right)\right)\right)+ \\
& \frac{1}{4} \gamma_{2}^{-1}\left(\mu V\left(x\left(h_{n}\right)\right)\right)
\end{aligned}
$$

over the domain of existence of the solution $x$.

The proof of the result is given in the Appendix.

Notice that

$$
\gamma_{2}(\|e(t)\|) \leq \gamma_{2}\left(2\left\|x\left(t_{k\left(h_{n}\right)}\right)\right\|\right)+\gamma_{2}(2\|x(t)\|)
$$

where we have exploited the inequality $\gamma_{2}(a+b) \leq \gamma_{2}(2 a)+$ $\gamma_{2}(2 b)$. From (15), we obtain

$$
\begin{gathered}
\gamma_{2}\left(2\left\|x\left(t_{k\left(h_{n}\right)}\right)\right\|\right) \leq \gamma_{2}\left(\frac{1}{2} \gamma_{2}^{-1}\left(\lambda(1-c) V\left(x\left(h_{n}\right)\right)\right)+\right. \\
\left.\frac{1}{2} \gamma_{2}^{-1}\left(\mu V\left(x\left(h_{n}\right)\right)\right)\right) \leq(\lambda(1-c)+\mu) V\left(x\left(h_{n}\right)\right)
\end{gathered}
$$

On the other hand

$$
\gamma_{2}(2\|x(t)\|) \leq \gamma_{2}\left(2 \alpha_{1}^{-1}(V(x(t)))\right) \leq \mu V(x(t))
$$

where the last inequality descends from (12).

Hence, for all $t \in H_{n}$, we have

$$
\begin{gathered}
\dot{V}(x(t)) \leq-\lambda V(x(t))+\gamma_{2}(\|e(t)\|) \\
\leq(\mu-\lambda) V(x(t))+(\lambda(1-c)+\mu) V\left(x\left(h_{n}\right)\right) .
\end{gathered}
$$

For those $t \in H_{n}$ such that $V\left(x\left(h_{n}\right)\right) \leq V(x(t))$,

$$
\dot{V}(x(t)) \leq(2 \mu-c \lambda) V(x(t))<\omega_{2} V(x(t))
$$

with $\omega_{2}:=\lambda(1-c)+2 \mu$, while for those $t \in H_{n}$ such that $V\left(x\left(h_{n}\right)\right)>V(x(t))$,

$$
\dot{V}(x(t)) \leq(\lambda(1-c)+2 \mu) V\left(x\left(h_{n}\right)\right)=\omega_{2} V\left(x\left(h_{n}\right)\right) .
$$

Inequalities (16) and (17) can be combined to prove the following:

Theorem 1 Consider the control system $\Sigma$ composed of (1) in closed-loop with (6), under DoS attacks satisfying Assumption 1, and with control update rule defined in (14). Let Assumption 2 hold.

If the parameter $\tau$ in (11) satisfies

$$
\tau>\frac{\omega_{1}}{\omega_{1}+\omega_{2}}=\frac{c \lambda}{\lambda+2 \mu},
$$

where

$$
\omega_{1}=c \lambda, \quad \omega_{2}=\lambda(1-c)+2 \mu,
$$

then any solution to $\Sigma$ satisfies the inequality

$$
\|x(t)\| \leq \alpha_{1}^{-1}\left(e^{\kappa\left(\omega_{1}+\omega_{2}\right)} e^{-\left[\omega_{1}-\left(\omega_{1}+\omega_{2}\right) / \tau\right] t} \alpha_{2}(\|x(0)\|)\right)
$$

for all $t \geq 0$.

We refer the reader to the Appendix for a proof of the theorem.

Remark 2 The result proves global asymptotic stability. In fact, the need to restrict the set of initial conditions within a ball of arbitrary large radius, thus obtaining a semi-global asymptotic stabilizability result, becomes apparent when we prove in Section $\mathrm{V}$ that the sequence of control update times generated by (14) occurs at a finite rate.

\section{DOS-INDUCED ACTUATION DELAY}

The analysis of the previous section rests upon the fulfillment of condition (14). In practice the sequence of control update times occurs at a finite sampling rate. This has a consequence on the actual duration of the DoS. As a matter of fact, when a sensor attempts to transmit and no acknowledgement is received due to the attack, it will repeat the transmission attempts until the transmission is successful. Due to the finite transmission rate, even when transmission becomes possible, there will be a delay from the time the DoS attack is over and the time the transmission can successfully occur. This delay causes a prolongation of the DoS interval that affects the stability result of the previous section. In this section, after recalling some notation from [5], [6], we provide a stabilization result that takes into account such a prolongation of the DoS attack. In other words, we remove the second simplifying assumption stated in ii) after (14).

Consider a control update sequence $\left\{t_{k}\right\}$ along with a DoS sequence $\left\{h_{n}\right\}$, and let

$$
\mathbb{S}_{n}:=\left\{k \in \mathbb{N} \mid t_{k} \in H_{n}\right\}
$$


denote the set of integers associated with an attempt to update the control action during $H_{n}$. Accordingly, by defining

$$
\Delta_{\mathbb{S}_{n}}:=\sup _{k \in \mathbb{S}_{n}} \Delta_{k}
$$

then

$$
\bar{H}_{n}:=\left[h_{n}, h_{n}+\tau_{n}+\Delta_{\mathbb{S}_{n}}[\right.
$$

will provide an upper bound on the $n$-th time interval over which the control action is not updated, while

$$
\bar{\Xi}(t):=\bigcup_{n \in \mathbb{N}} \bar{H}_{n} \bigcap[0, t]
$$

will provide an upper bound on the total interval up to the current time over which the control action is not updated. Equation (21) essentially models the additional delay in the control update that may arise under finite sampling rate.

We are now ready to state a version of Theorem 1 in which the DoS-attack-induced delay is taken into account.

Theorem 2 Consider the control system $\Sigma$ composed of (1) in closed-loop with (6), under DoS attacks satisfying Assumption 1, and with control update rule defined in (14). Let Assumption 2 hold.

If the parameter $\tau$ in (11) satisfies

$$
\tau>\frac{c \lambda}{\lambda+2 \mu}\left(1+\frac{\Delta_{*}}{\tau_{*}}\right)
$$

where

$$
\Delta_{*}:=\sup _{n \in \mathbb{N}} \Delta_{\mathbb{S}_{n}}
$$

and

$$
\tau_{*}:=\inf _{n \in \mathbb{N}} \tau_{n}>0,
$$

then the inequality

$$
\|x(t)\| \leq \alpha_{1}^{-1}\left(\gamma \mathrm{e}^{-\beta t} \alpha_{2}(\|x(0)\|)\right)
$$

holds for all $t \geq 0$, where

$$
\gamma=e^{\kappa(\lambda+2 \mu)\left(1+\frac{\Delta_{*}}{\tau_{*}}\right)}, \quad \beta=\left(c \lambda-\frac{\lambda+2 \mu}{\tau}\left(1+\frac{\Delta_{*}}{\tau_{*}}\right)\right)
$$

and the parameters $\kappa, \lambda, \mu$, are as in (11), (2) and (12), respectively.

Proof. For any $t \in \mathbb{R}_{\geq 0}$, the Lyapunov function evolves as $\dot{V}(x(s)) \leq-\omega_{1} V(x(s))$ if $s \notin \bar{\Xi}(t)$ and as $\dot{V}(x(s)) \leq$ $\omega_{2} V(x(s))$ if $s \in \bar{\Xi}(t)$. Hence

$$
V(x(t)) \leq \mathrm{e}^{-\omega_{1} t+\left(\omega_{1}+\omega_{2}\right)|\bar{\Xi}(t)|} V\left(x\left(t_{0}\right)\right) .
$$

In [6], Theorem 2, the following estimate of $|\bar{\Xi}(t)|$ is provided:

$$
|\bar{\Xi}(t)| \leq\left(\kappa+\frac{t}{\tau}\right)\left(1+\frac{\Delta_{*}}{\tau_{*}}\right)
$$

Hence,

$$
V(x(t)) \leq \mathrm{e}^{-\left(\omega_{1}-\frac{\omega_{1}+\omega_{2}}{\tau}\left(1+\frac{\Delta_{*}}{\tau_{*}}\right)\right) t+\kappa\left(\omega_{1}+\omega_{2}\right)\left(1+\frac{\Delta_{*}}{\tau_{*}}\right)} V\left(x\left(t_{0}\right)\right) .
$$

The thesis now follows immediately under the stated assumptions and bearing in mind that $\omega_{1}=c \lambda, \omega_{2}=\lambda(1-c)+2 \mu$.

Remark 3 Theorem 2 differs from Theorem 1 not only because of $\Delta_{*}$ but also due to the presence of $\tau_{*}$. This has a very intuitive explanation. In fact, in the ideal case considered in Theorem $1, \Delta_{*}=0$ since a control update can always occur as soon as DoS is over. Under finite sampling rate, each DoS interval will instead possibly introduce an additional delay in the control update. This also points out that, given two DoS sequences of equal total length, the one composed of more intervals having smaller duration will be more critical for stability, since it will potentially deny more communications attempts.

\section{Finite SAMPLing RATE}

Until now in the investigation we have been implicitly assuming that the sampling sequence generated by the rule (14) occur at a finite sampling rate. In this section we investigate conditions under which such assumption is actually met in practice. To this end, we restrict the set of initial conditions for the process. In fact, we say that the solutions to the control system $\Sigma$ composed of (1) in closed-loop with (6), under DoS attacks satisfying Assumption 1, and with control update rule defined in (14), have ([16]) a semi-global uniform finite sampling rate if for every $R>0$ and for every DoS sequence satisfying Assumption 1, there exists $\varepsilon_{R}>0$ such that any solution to $\Sigma$ starting in the ball $B_{R}(0)$ of radius $R$ and center the origin has an associated control update sequence $\left\{t_{k}\right\}$ that for all $k \in \mathbb{N}$ satisfies (9) with $\varepsilon$ replaced by $\varepsilon_{R}>0$.

Let $R$ be an arbitrary positive number such that $\|x(0)\| \mid \leq$ $R$. Motivated by the bound in (34), we introduce the balls

$\mathcal{X}=B_{\alpha_{1}^{-1}\left(\gamma \alpha_{2}(R)\right)}(0) \subset \mathbb{R}^{n}, \mathcal{E}=B_{2 \alpha_{1}^{-1}\left(\gamma \alpha_{2}(R)\right)}(0) \subset \mathbb{R}^{n}$.

Notice that these balls are known a priori since their radius depend on quantities that are fixed in advance.

Assume that the vector field $f(x, k(x+e))$ is locally Lipschitz with respect to the variables $(x, e)$, and let $L$ be the Lipschitz constant such that

$\|f(x, k(x+e))\| \leq L(\|x\|+\|e\|)$, for all $(x, e) \in \mathcal{X} \times \mathcal{E}$.

The first fact that we recall is that as far as $x \in \mathcal{X}$, then $e \in \mathcal{E}$. Therefore, denoted by $\sigma$ the positive constant whose inverse is the Lipschitz constant of the function $\alpha_{1}^{-1}\left(\frac{1}{\lambda(1-c)} \gamma_{2}(4\|e\|)\right)$ on $\mathcal{E}$ (under the assumption that the function is locally Lipschitz), we have that

$$
\alpha_{1}^{-1}\left(\frac{1}{\lambda(1-c)} \gamma_{2}(4\|e\|)\right) \leq \frac{1}{\sigma}\|e\|, \quad e \in \mathcal{E} .
$$

Hence, as far as $x(t) \in \mathcal{X}$, any sequence $\left\{t_{k}\right\}$ generated by the triggering rule

$$
\|e(t)\| \leq \sigma\|x(t)\|, \quad t \in\left[t_{k}, t_{k+1}\right]
$$


implies that (14) is satisfied as well, that is

$$
\gamma_{2}(4\|e(t)\|) \leq \lambda(1-c)\|x(t)\|, \quad t \in\left[t_{k}, t_{k+1}\right] .
$$

Now we prove that with a triggering rule as in (30), the requirement $t_{k+1}-t_{k} \geq \varepsilon$ for all $k \in \mathbb{N}$ is actually met. To show this, we study the evolution of $e(t)$ for $t \in\left[t_{k}, t_{k+1}[\right.$. Observe that

$$
\dot{e}(t)=-f(x, k(x+e))
$$

implies

$$
e(t) \leq-\int_{t_{k}}^{t} f(x(s), k(x(s)+e(s))) d s
$$

and hence

$$
\|e(t)\| \leq \int_{t_{k}}^{t} L(\|x(s)\|+\|e(s)\|) d s .
$$

Since $\|x(s)\| \leq\left\|x\left(t_{k}\right)\right\|+\|e(s)\|$, we also have

$$
\|e(t)\| \leq L\left\|x\left(t_{k}\right)\right\|\left(t-t_{k}\right)+\int_{t_{k}}^{t} 2 L\|e(s)\| d s .
$$

Applying Gronwall-Bellman's inequality and after standard but lengthy manipulations, one arrives at

$$
\|e(t)\| \leq \frac{1}{2}\left(\mathrm{e}^{2 L\left(t-t_{k}\right)}-1\right)\left\|x\left(t_{k}\right)\right\| .
$$

Let $g(\tau):=\frac{1}{2}\left(\mathrm{e}^{2 L \tau}-1\right)$. Since $\left\|x\left(t_{k}\right)\right\| \leq\|e(t)\|+\|x(t)\|$, for all $t \geq t_{k}$ such that $g\left(t-t_{k}\right)<1$, then the previous inequality is equivalent to

$$
\|e(t)\| \leq \frac{g\left(t-t_{k}\right)}{1-g\left(t-t_{k}\right)}\|x(t)\| .
$$

Now,

$$
g\left(t-t_{k}\right)<1 \text { and } \frac{g\left(t-t_{k}\right)}{1-g\left(t-t_{k}\right)} \leq \sigma
$$

if and only if

$$
\mathrm{e}^{2 L\left(t-t_{k}\right)}<\min \left\{3, \frac{3 \sigma+1}{\sigma+1}\right\}
$$

or equivalently

$$
t-t_{k}<\frac{1}{2 L} \ln \left(\frac{3 \sigma+1}{\sigma+1}\right) .
$$

Thus condition (30) is not violated until at least

$$
\frac{1}{2 L} \ln \left(\frac{3 \sigma+1}{\sigma+1}\right)
$$

units of time have elapsed. In other words, any sequence of sampling times generated by the triggering rule (30) or (14) occurs at a finite sampling rate, that is (9) holds, with $\varepsilon$ replaced by $\varepsilon_{R}$ and $\varepsilon_{R}=\frac{1}{2 L} \ln \left(\frac{3 \sigma+1}{\sigma+1}\right)$.

The discussion allows us to draw the following conclusion:

Theorem 3 Consider system (1) in closed loop with the control (6), under DoS attacks satisfying Assumption 1, and with control update rule defined in (14). Let Assumption 2 hold. Then, given any $R>0$, if the parameter $\tau$ in (11) satisfies

$$
\tau>\frac{c \lambda}{\lambda+2 \mu}\left(1+\frac{\Delta_{*}}{\tau_{*}}\right)
$$

where

$$
\Delta_{*}:=\sup _{n \in \mathbb{N}} \Delta_{\mathbb{S}_{n}}
$$

and

$$
\tau_{*}:=\inf _{n \in \mathbb{N}} \tau_{n}>0
$$

then the inequality

$$
\|x(t)\| \leq \alpha_{1}^{-1}\left(\gamma \mathrm{e}^{-\beta t} \alpha_{2}(\|x(0)\|)\right)
$$

holds for all $t \geq 0$ and for all $\|x(0)\| \leq R$, where

$$
\gamma=e^{\kappa(\lambda+2 \mu)\left(1+\frac{\Delta_{*}}{\tau_{*}}\right)}, \quad \beta=\left(c \lambda-\frac{\lambda+2 \mu}{\tau}\left(1+\frac{\Delta_{*}}{\tau_{*}}\right)\right),
$$

and the parameters $\kappa, \lambda, \mu$, are as in (11), (2), (12), respectively. Moreover, the control update rule has a semi-global uniform finite sampling rate and (9) holds for each $k \in \mathbb{N}$ with with $\varepsilon$ replaced by $\varepsilon_{R}$ and $\varepsilon_{R}=\frac{1}{2 L} \ln \left(\frac{3 \sigma+1}{\sigma+1}\right)$.

There is a clear trade-off between the sampling rate and the DoS attacks that the system can tolerate. In fact, the minimum inter-sampling time $\varepsilon_{R}$ depends on both $\sigma$ and $L$. In turn, these parameters depend on the radius of the compact set $\mathcal{X}$. This radius is affected by the parameter $\gamma$ : the larger is $\gamma$ the larger is the radius. Now, $\gamma$ grows with $\kappa$ that appears in the description of the DoS signals, modeling possible occurrence of large intervals of DoS attacks at initial times. Thus, a sustained attack at initial times might lead to a small minimum inter sampling-time. On the other hand the parameter $\tau$ that accounts for the percentage of time for which an attack takes place depend on both the data of the nonlinear system under control and the characteristic of the DoS attack signal.

The result gives an indication on how to implement event-triggered control to stabilize nonlinear systems in the presence of DoS attacks. Event-triggered control requires continuous monitoring of the state $x(t)$ and can be resourceconsuming. Alternative implementations in the spirit of Section 4.2 in [6] will be investigated in future versions of this work.

\section{CONCLUSIONS}

The paper investigates the design of event-based control strategies for nonlinear systems in the presence of DoS attacks that interrupts the flow of information from the sensors to the actuators. The DoS signal attack is modeled at a fairly general level that we believe allows for the inclusion of several interesting scenarios. Relations between the sampling frequency, the data of the nonlinear systems under control and the features of the DoS attack signal have been revealed.

The main working assumption is Assumption 2 whose role is to prevent the occurrence of finite escape times. It therefore 
restricts the class of nonlinear systems but allows for a less complicated analysis. Clearly, removing this assumption requires to restrict the class of DoS attacks the system can tolerate: if the systems undergoes prolonged attacks, it will evolve in open loop for long time intervals facing the possible occurrence of a finite escape time. This alternative formulation may be worth of investigation.

In future work, more attention will be given to the actual implementation of our resilient control and in particular to its connections with other event-based approaches such as self-triggered control. Relevant case studies to assess the effectiveness of our approach are also part of our future research plan. Whether our method can deal with attack scenarios different from DoS attacks is a topic worth of investigation as well.

Robustness of the proposed resilient control (as well as of its linear counterpart studied in [5], [6]) to external disturbances in an ISS sense is a very interesting and challenging research topic that will be tackled in the future.

Our initial interest for resilient control was motivated by distributed control strategies for dynamical networks in a cyberphysical environment (see e.g. [4]). We believe that our technique can be extended to distributed resilient control and can be a very fertile research ground.

\section{APPENDIX}

Proof of Lemma 1 Recall that $e(t)=x\left(t_{k\left(h_{n}\right)}\right)-x(t)$ by definition, so that

$$
\begin{gathered}
\left\|x\left(t_{k\left(h_{n}\right)}\right)\right\|-\left\|x\left(h_{n}\right)\right\| \leq\left\|e\left(h_{n}\right)\right\| \\
\quad \leq \frac{1}{4} \gamma_{2}^{-1}\left(\lambda(1-c) V\left(x\left(h_{n}\right)\right)\right)
\end{gathered}
$$

where the second inequality descends from the control update rule (14). Hence

$$
\left\|x\left(t_{k\left(h_{n}\right)}\right)\right\| \leq \frac{1}{4} \gamma_{2}^{-1}\left(\lambda(1-c) V\left(x\left(h_{n}\right)\right)\right)+\left\|x\left(h_{n}\right)\right\| .
$$

On the other hand

$$
\left\|x\left(h_{n}\right)\right\| \leq \alpha_{1}^{-1}\left(V\left(x\left(h_{n}\right)\right)\right) \leq \frac{1}{4} \gamma_{2}^{-1}\left(\mu V\left(x\left(h_{n}\right)\right)\right),
$$

where the second inequality is implied by condition (12). Combining the two inequalities above the thesis descend.

Proof of Theorem 1 For any $t \in \mathbb{R}_{>0}$, the Lyapunov function evolves as $\dot{V}(x(s)) \leq-\omega_{1} V(x(s))$ if $s \notin \Xi(t)$ and $\dot{V}(x(s)) \leq \omega_{2} V(x(s))$ if $s \in \Xi(t)$. Hence

$$
\begin{aligned}
V(x(t)) & \leq \mathrm{e}^{-\omega_{1}(t-|\Xi(t)|)} \mathrm{e}^{\omega_{2}|\Xi(t)|} V\left(x\left(t_{0}\right)\right) \\
& \leq \mathrm{e}^{-\omega_{1} t+\left(\omega_{1}+\omega_{2}\right)|\Xi(t)|} V\left(x\left(t_{0}\right)\right) .
\end{aligned}
$$

Bearing in mind Assumption 1, and in particular condition (11), it follows that

$$
V(x(t)) \leq \mathrm{e}^{-\left(\omega_{1}-\left(\omega_{1}+\omega_{2}\right) \tau\right) t+\kappa\left(\omega_{1}+\omega_{2}\right)} V\left(x\left(t_{0}\right)\right) .
$$

The thesis now follows immediately under the stated assumptions.

\section{REFERENCES}

[1] S. Amin, A. Càrdenas, and S.S. Sastry. Safe and secure networked control systems under denial of-service attacks. In Hybrid systems: Computation and Control, pages 31-45, 2009.

[2] W. Bian and M. French. General fast sampling theorems for nonlinear systems. Systems \& Control Letters, 54:1037-1050, 2005.

[3] E. Byres and J. Lowe. The myths and facts behind cyber security risks for industrial control systems. Proceedings of the VDE Congress, Berlin, 2004.

[4] C. De Persis and P. Frasca. Robust self-triggered coordination with ternary controllers. IEEE Transactions on Automatic Control, 58(12):3024-3038, 2013.

[5] C. De Persis and P. Tesi. Resilient control under denial-of-service. In Proceedings of the IFAC World Conference, Cape Town, South Africa.

[6] C. De Persis and P. Tesi. Resilient control under denial-of-service. arXiv:1311.5143, 2013.

[7] H. Shisheh Foroush and S. Martínez. On triggering control of single-input linear systems under pulse-width modulated dos jamming attacks. International Journal of Robust and Nonlinear Control, 2013. Submitted.

[8] H. Shisheh Foroush and S. Martínez. On event-triggered control of linear systems under periodic denial of service attacks. Proc. of the IEEE Conf. on Decision and Control, Maui, HI, USA, 2012.

[9] R.A. Freeman and P.V. Kokotovic. Inverse optimality in robust stabilization. SIAM J. Control, 34:1365-1391, 1996.

[10] A. Gupta, C. Langbort, and T. Başar. Optimal control in the presence of an intelligent jammer with limited actions. Proc. of the 49th IEEE CDC, Atlanta, GA, USA, 2010.

[11] D. Liberzon, E.D. Sontag, and Y. Wang. Universal construction of feedback laws achieving iss and integral iss disturbance attenuation. Systems \& Control Letters, 46:111-127, 2002.

[12] Y. Mo, T. Hyun-Jin Kim, K. Brancik, D. Dickinson, H. Lee, A. Perrig, and Bruno Sinopoli. Cyber-physical security of a smart grid infrastructure. Proceedings of the IEEE, 100:195-209, 2012.

[13] D. Nešić and D.S. Laila. A note on input-to-state stabilization for nonlinear sampled-data systems. IEEE Trans. Aut. Contr., 47:1153$1158,2002$.

[14] D. Nešić and A.R. Teel. A framework for stabilization of nonlinear sampled-data systems based on their approximate discrete-time models. IEEE Trans. Aut. Contr., 49:1103-1122, 2004.

[15] D. Nešić, A.R. Teel, and P.V. Kokotovic. Sufficient conditions for stabilization of sampled-data nonlinear systems via discrete-time approximations. Systems \& Control Letters, 38:259-270, 1999.

[16] R. Postoyan, P. Tabuada, D. Nešić, and A. Anta. Event-triggered and self-triggered stabilization of networked control systems. In IEEE Conf. on Decision and Control and European Control Conf., Orlando, U.S.A., pages 2565-2570, 2011.

[17] L. Schenato, B. Sinopoli, M. Franceschetti, K. Poolla, and S.S Sastry. Foundations of control and estimation over lossy networks. Proceedings of the IEEE, 95:163-185, 2007.

[18] G. Seyboth, D. Dimarogonas, and K. Johansson. Event-based broadcasting for multi-agent average consensus. Automatica, 49:245-252, 2013.

[19] P. Tabuada. Event-triggered real-time scheduling of stabilizing control tasks. IEEE Trans. Aut. Contr., 52:1680-1685, 2007.

[20] J. Tsinias. Control lyapunov functions, input-to-state stability and applications to global feedback stabilization for composite systems. $J$. Math. Systems Estimate Control, 7:1-31, 1997.

[21] L. Vu and D. Liberzon. Input-to-state stability of switched systems and switching adaptive control. Automatica, 43:639-646, 2007. 